\title{
Balance impairment and systemic inflammation in chronic obstructive pulmonary disease
}

\author{
This article was published in the following Dove Press journal: \\ International Journal of COPD \\ 8 September 2015 \\ Number of times this article has been viewed
}

\section{Emanuela Tudorache' \\ Cristian Oancea' \\ Claudiu Avram ${ }^{2}$ \\ Ovidiu Fira-Mladinescu' \\ Lucian Petrescu ${ }^{3}$ \\ Bogdan Timar ${ }^{4}$}

'Department of Pulmonology, University of Medicine and Pharmacy "Victor Babes", ${ }^{2}$ Physical Education and Sport Faculty, West University of Timisoara, ${ }^{3}$ Department of Cardiology, University of Medicine and Pharmacy "Victor Babes", ${ }^{4}$ Department of Biostatistics and Medical Informatics, University of Medicine and Pharmacy "Victor Babes", Timisoara, Romania

Correspondence: Cristian Oancea Department of Pulmonology, University of Medicine and Pharmacy "Victor Babes”, Eftimie Murgu Square, Number 2, Timisoara, Romania

Tel +40 769221057

Fax +40 256207673

Email oancea@umft.ro
Background/purpose: Chronic obstructive pulmonary disease (COPD), especially in severe forms, is commonly associated with systemic inflammation and balance impairment. The aim of our study was to evaluate the impact on equilibrium of stable and exacerbation (acute exacerbation of COPD [AECOPD]) phases of COPD and to investigate if there is a connection between lower extremity muscle weakness and systemic inflammation.

Methods: We enrolled 41 patients with COPD (22 stable and 19 in AECOPD) and 20 healthy subjects (control group), having no significant differences regarding the anthropometric data. We analyzed the differences in balance tests scores: Falls Efficacy Scale-International (FES-I) questionnaire, Berg Balance Scale (BBS), Timed Up and Go (TUG) test, Single Leg Stance (SLS), 6-minute walking distance (6MWD), isometric knee extension (IKE) between these groups, and also the correlation between these scores and inflammatory biomarkers.

Results: The presence and severity of COPD was associated with significantly decreased score in IKE $(P<0.001), 6 \mathrm{MWD}(P<0.001)$, SLS $(P<0.001)$, and BBS $(P<0.001)$, at the same time noting a significant increase in median TUG score across the studied groups $(P<0.001)$. The AECOPD group vs stable group presented a significant increase in high-sensitive C-reactive protein (hs-CRP) levels (10.60 vs 4.01; $P=0.003$ ) and decrease in $\mathrm{PaO}_{2}(70.1$ vs $59.1 ; P<0.001)$. We observed that both IKE scores were significantly and positive correlated with all the respiratory volumes. In both COPD groups, we observed that fibrinogen reversely and significantly correlated with the 6MWD, and FES-I questionnaire is correlated positively with TUG test. Hs-CRP correlated reversely with the walking test and SLS test, while correlating positively with TUG test and FES-I questionnaire.

Conclusion: According to this study, COPD in advanced and acute stages is associated with an increased history of falls, systemic inflammation, balance impairment, and lower extremity muscle weakness.

Keywords: balance, COPD, inflammation, muscle, falls

\section{Introduction}

Chronic obstructive pulmonary disease (COPD) is an inflammatory disorder characterized by progressive airflow limitation. ${ }^{1}$ Until a few years ago, the traditional treatment only focused on lung function, but recently, systemic manifestations are receiving increased attention. Although reduction in muscle performance, functional mobility, and exercise capacity has been demonstrated, more evidence suggests that individuals with COPD also present an important risk of falls. ${ }^{2}$

Multiple medications, impaired mobility, muscle weakness, and the presence of chronic diseases are often mentioned as risk factors that can lead to falls in older adults. ${ }^{3}$ Just a small percentage of falls are the result of a single cause; therefore, the etiology is considered to be multifactorial, involving patient-related factors (intrinsic) and environmental circumstances (extrinsic) as well as variables related with the activity. ${ }^{4}$ 
The most cited intrinsic factors are dizziness followed by lower extremity muscle weakness. Lower extremity muscle weakness produces up to $26 \%$ of the institutional falls and $13 \%$ of the community falls. ${ }^{5}$

Skeletal muscle dysfunction is one of the important extrapulmonary manifestations of COPD. Hypoxemia, malnutrition, disuse, and systemic inflammation in skeletal muscle are several factors that induce changes in the muscle mass structure and function in COPD. ${ }^{6}$ Along with the increasing severity of the disease, COPD patients lose muscle mass, especially in their thighs. ${ }^{7}$ As a consequence, patients will accuse decreased exercise endurance, fatigue, and dyspnea at a minimal degree of exertion. These symptoms will reduce their ability to perform daily activities and limit their exercise tolerance, creating a downward spiral that will lead to generalized immobility. ${ }^{8}$ Lower extremity muscle weakness being an important extrapulmonary manifestation and a significant risk factor for falls, we can presume that COPD population have a higher risk for falls than their healthy peers.

\section{Study aim}

The objective of this study was to investigate if COPD patients especially in acute stages have balance impairment and if there is a connection between lower extremity muscle weakness and systemic inflammation.

\section{Materials and methods Study design}

Before participating in the study, all patients signed an informed consent form. The study was approved by the ethical board of the "Victor Babes" Hospital for Infectious and Lung Diseases, Timisoara, Romania.

\section{Subjects}

In this study, we included 61 individuals, with a median age of 60 years (interquartile range 4 years) and an average body mass index (BMI) of $24.8 \pm 4.8 \mathrm{~kg} / \mathrm{m}^{2}$. Subjects were divided into three study groups: the control group (Group A) that included healthy subjects, Group B with stable COPD patients (patients who have clinically stable airway obstruction and did not require any change in therapeutic treatment plan in the last 3 months), and Group C with AECOPD patients (according to Global Initiative for Chronic Obstructive Lung Disease guidelines). The baseline characteristics of the three cohorts are presented in Table 1. No significant differences regarding age or BMI were observed between the three groups. None of our patients were on long-term oxygen therapy.

Inclusion criteria for the COPD (22 stable, 19 acute exacerbation patients) groups were: history of smoking $>20$ packs-year, physician diagnosis of COPD (forced expiratory volume in 1 second $\left[\mathrm{FEV}_{1}\right]<80 \%$ predicted, $\mathrm{FEV}_{1} /$ forced vital capacity $[\mathrm{FVC}]<0.7$ predicted) according to the international guideline and the ability to provide inform consent. ${ }^{9}$ We included in the AECOPD group only exacerbations that did not require admission to ICU.

The control group included 20 subjects, age and sex matched with normal spirometry $\left(\mathrm{FEV}_{1} \geq 80 \%\right.$ predicted, $\mathrm{FEV}_{1} / \mathrm{FVC} \geq 0.7$ ) with the absence of any health-related problems that could impair balance and mobility.

Exclusion criteria: syncope, known cognitive impairment, use of medication that may have increased the risk of falls (eg, psychotropic drugs), postural orthostatic hypotension, neurological or musculoskeletal diseases that could account for possible falls and imbalance (eg, Parkinson's disease), history of cerebral-vascular accident, severe cardiovascular disease, transient ischemic attacks, or lower extremity joint replacements.

\section{Protocol and equipment}

On admission day, blood samples, gases, and inflammatory biomarkers (fibrinogen and high-sensitive C-reactive protein [hs-CRP]) were collected from all subjects. Pulmonary

Table I Patient's baseline characteristics

\begin{tabular}{|c|c|c|c|c|}
\hline Characteristics & Control group & Stable COPD group & AECOPD group & $P$-value \\
\hline Age (years) $)^{\S}$ & $63(4)$ & $63(5)$ & $63(3)$ & 0.829 \\
\hline $\mathrm{BMI}\left(\mathrm{kg} / \mathrm{m}^{2}\right)$, mean $\pm \mathrm{SD}^{¥}$ & $24.9 \pm 3.8$ & $24.2 \pm 5.7$ & $25.1 \pm 4.8$ & 0.824 \\
\hline History of falls (\%) & 26 & 39 & 43 & $<0.00 I^{*}$ \\
\hline $\mathrm{FVC}(\mathrm{L})^{\S}$ & $4.75(0.65)$ & $2.68(0.98)$ & $2.34(0.61)$ & $<0.00 I^{*}$ \\
\hline $\operatorname{FVC~}(\%)^{\S}$ & $101(10)$ & $65.5(80)$ & $66(4)$ & $<0.00 I^{*}$ \\
\hline $\mathrm{FEV}_{1}(\mathrm{~L})^{\S}$ & $3.57(0.65)$ & $0.79(0.36)$ & $0.7 I(0.3 I)$ & $<0.00 I^{*}$ \\
\hline $\mathrm{FEV}_{1}(\%)^{\S}$ & $100.5(12)$ & $27.5(7)$ & $20.0(10)$ & $<0.00 I^{*}$ \\
\hline $\mathrm{FEV}_{1} / \mathrm{FVC}^{\S}$ & $76(10)$ & $27.6(8)$ & $31.8(10)$ & $<0.00 I^{*}$ \\
\hline $\mathrm{PaO}_{2}(\mathrm{mmHg})$, mean $\pm \mathrm{SD}^{*}$ & Not performed & $70.1 \pm 9.1$ & $59.1 \pm 8.9$ & $<0.00 I^{*}$ \\
\hline
\end{tabular}

Notes: ${ }^{\complement} \bigvee$ ariables without Gaussian distribution; $P$-value was calculated using Kruskal-Wallis test. ${ }^{*} V$ ariables with Gaussian distribution; $P$-value was calculated using one-way ANOVA test. Data is presented as median (interquartile range), unless otherwise noted. *Differences are significant.

Abbreviations: AECOPD, acute exacerbation of COPD; ANOVA, analysis of variance; BMI, body mass index; FVC, forced vital capacity; FEV ${ }_{1}$, forced expiratory volume in I second. 
function tests were obtained through spirometry. Subjects completed the Falls Efficacy Scale-International (FES-I) questionnaire and performed the balance tests on the 1st day of admission. All tests were conducted by the same physiotherapist.

\section{Balance measures}

Patients completed the FES-I questionnaire that assesses the confidence level individuals have in performing daily activities without falling. This tool was developed to expand Tinetti's initial FES and includes social activities that may be considered more challenging by active people, thus potentially causing more concerns about falling and then the basic activities represented in the initial questionnaire. It is a 16-point questionnaire on which the level of concern is measured on a 4-point scale ( $1=$ not at all concerned to $4=$ very concerned). ${ }^{10}$ The first balance test performed by the patients was the Berg Balance Scale (BBS). This test measures the functional balance using 14 items. Activities such as reaching, transfers, and turning around were graded on a scale ranging from 0 (unable/unsafe) to 4 (independent/ safe), ${ }^{11}$ with higher scores indicating greater balance control. A cutoff score of 46 and below has been identified as a useful score to successfully identify those at risk of falling. Afterward, the patients performed the Timed Up and Go (TUG) test which is a timed performance measure that includes balance and gait maneuvers used in activities of daily living and Single Leg Stance (SLS), which is a task of static balance that records the time the participant is able to stand on one leg unassisted. Throughout the assessment session, participants were encouraged to rest as needed. The SLS and TUG tests were performed three times, and the best value was recorded. ${ }^{12}$ A cutoff score of 16 or more in TUG test has been shown to predict falls in community-dwelling elderly. ${ }^{2}$ To assess the functional exercise level, we performed the 6-minute walking distance (6MWD) according to the American Thoracic Society guidelines. ${ }^{13}$

Isometric knee extension (IKE) test was measured using a digital handheld dynamometer (Chatillon K-FCE-200, USA). Participants were seated upright on the testing chair with the knee bent at $\sim 90^{\circ}$ with the lower leg hanging down the front of the chair. Knee extension was tested three times on each leg, with a 60 -second rest period between each test. All the test values were recorded, but only the highest value of the three tests was used. ${ }^{14}$

A spirometer (Jaeger, Germany) was used. The FVC, $\mathrm{FEV}_{1}$, and the ratio $\mathrm{FEV}_{1} / \mathrm{FVC}$ were measured three times, and the best value was reported (according to the American Thoracic Society/European Respiratory Society statement) (Table 1).

\section{Statistical analysis}

Data were collected and analyzed using the SPSS Version 17 software suite (SPSS Inc., Chicago, IL, USA) and are presented as medians and interquartile range for continuous variables without Gaussian distribution and means \pm standard deviation for continuous variables with Gaussian distribution.

To assess the significance of the differences between groups, Mann-Whitney $U$ and Kruskal-Wallis tests (medians, non-Gaussian populations), analysis of variance, and unpaired Student's $t$-tests (means, Gaussian populations) were used. Continuous variable distributions were tested for normality using Shapiro-Wilk test. The correlation between studied variables was evaluated using Spearman's rank sum correlation coefficient (non-Gaussian distributed variables), its statistical significance being assessed using the $t$-distribution score test. In this study, a $P$-value of $<0.05$ was considered as the threshold for statistical significance.

\section{Results}

The presence and severity of COPD was associated with significantly decreased IKE score (Figures 1 and 2 ) in both left $(P<0.001)$ and right $(P<0.001)$, decreased score in walking test $(P<0.001)$, SLS $(P<0.001)$, and BBS score $(P<0.001)$, at the same time noting significant increases in median TUG score across the studied groups $(P<0.001)$. The comparison of these parameters is presented in Table 2 . Inflammatory markers and $\mathrm{PaO}_{2}$ were measured only for patients with COPD and AECOPD. While the increases in fibrinogen values associated with AECOPD had no statistical significance, this group was associated with a significant increase in hs-CRP levels (10.60 vs $4.01 ; P=0.003) . \mathrm{PaO}_{2}$ was significantly decreased in AECOPD $(P<0.001)$.

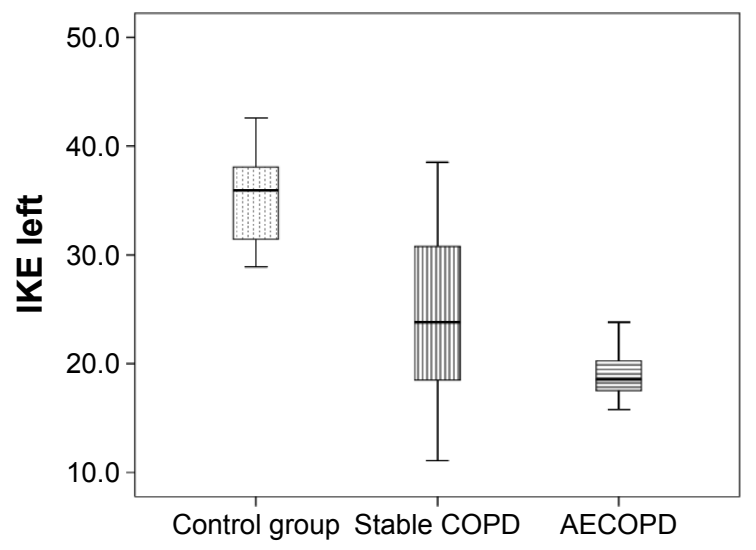

Figure I Boxplot for distribution of IKE score in the left leg across the studied cohorts.

Abbreviations: AECOPD, acute exacerbation of COPD; IKE, isometric knee extension. 


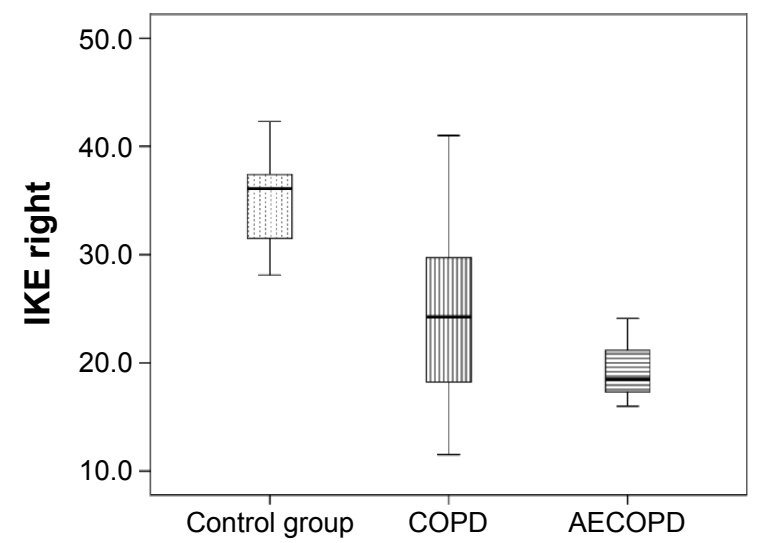

Figure 2 Boxplot graph for distribution of IKE score in the right leg across the studied cohorts.

Abbreviations: AECOPD, acute exacerbation of COPD; IKE, isometric knee extension.

We observed that both IKE scores measured in left and right leg, respectively, were significantly and positively correlated with all the respiratory volumes; an observation which points out that the strength of quadriceps decreases along with the pulmonary impairment (Table 3).

In both COPD groups, we observed that fibrinogen reversely and significantly correlated with walking test score and FES, respectively, and correlated positively with TUG. Hs-CRP correlated reversely with the walking test and SLS, while correlated positively with TUG and FES. The correlations between inflammatory tests and the other studied balance and strength parameters had no statistical significance (Table 4).

We divided our groups in age-based subgroups (60-64 years, 65-69 years, 70-74 years) but we did not record an increased number of falls related to age. The number of falls in COPD groups vs control group was significantly higher: $43 \%$ in AECOPD and 39\% in stable COPD, vs control group (26\%).

All the patients received inhaled bronchodilator therapy (in different associations), and $37 \%$ of them received inhaled corticosteroids. In exacerbation, all the patients received systemic corticotherapy.

\section{Discussion}

Our results suggest that the presence of COPD (stable or exacerbation phases) is associated with: 1) an increased history of falls; 2) systemic inflammation (increased hs-CRP and fibrinogen levels, especially in AECOPD); 3) balance impairment (decreased SLS and BBS scores and increased FES and TUG scores); 4) lower extremity muscle weakness (decreased IKE score in both legs); and 5) a decline in physical activity capacity (decreased 6MWD score).

The novelty of this article is the study design. Previous papers have associated balance tests with pulmonary function tests, but this is the first study, to our knowledge, that also associates inflammatory biomarkers (hs-CRP and fibrinogen) and compares healthy subjects with stable and acute phases of COPD. We also used the FES-I questionnaire to self-assess the confidence level individuals have in performing daily activities without falling. The other studies used Activitiesspecific Balance Confidence (ABC) score with comparable results. ${ }^{2}$ In literature there are several risk factors, such as: aging, hypoxemia, medication, systemic inflammation, cognitive impairment, and disuse, etc, which can lead to falls in this population, and they will be discussed later., ${ }^{6} 15-17$

COPD population tends to be more sedentary, and another factor of risk for falls could be disuse. In the present study, we used 6MWD test to evaluate the exercise functional level and we recorded a significant difference between the COPD groups and the control group.

We have observed a reverse correlation between $\mathrm{FEV}_{1}$ and hs-CRP, highlighting the importance of systemic inflammation in relation with COPD status. On the other hand, inflammation biomarkers are associated with decreased muscle strength (IKE), exercise endurance (6MWD), and balance

Table 2 Comparison of IKE scores and balance parameters regarding the patients COPD status

\begin{tabular}{|c|c|c|c|c|}
\hline & Control group & Stable COPD group & AECOPD group & $P$-value \\
\hline IKE left $(\mathrm{kg})^{\S}$ & $35.9(7.1)$ & $23.8(13.1)$ & I8.5 (2.8) & $<0.00 I^{*}$ \\
\hline IKE right $(\mathrm{kg})^{\S}$ & $36.1(6.4)$ & $24.3(12.3)$ & I8.5 (3.9) & $<0.00 I^{*}$ \\
\hline 6MWD $(m)^{\S}$ & $431(42)$ & $363(67)$ & $266(85)$ & $<0.00 I^{*}$ \\
\hline $\operatorname{SLS}(s)^{\S}$ & $26(3.1)$ & $16.3(2.1)$ & $7.8(1.8)$ & $<0.00 I^{*}$ \\
\hline TUG $(s)^{\S}$ & $9.3(2.1)$ & $15.2(1.1)$ & I8.3 (2.6) & $<0.00 I^{*}$ \\
\hline BBS $(p t s)^{\S}$ & $53(5)$ & $46(3)$ & $42(4)$ & $<0.00 I^{*}$ \\
\hline FES $(p t s)^{\S}$ & $17.5(5)$ & $40(4)$ & $51(6)$ & $<0.00 I^{*}$ \\
\hline Fibrinogen $(g / L)$, mean $\pm S D^{*}$ & $2.62 \pm 0.4$ & $3.03 \pm 0.7$ & $5.84 \pm 6.2$ & $0.010 *$ \\
\hline $\mathrm{hs}-\mathrm{CRP}(\mathrm{mg} / \mathrm{L})$, mean $\pm \mathrm{SD}^{*}$ & $3.2 \pm 1.4$ & $4.01 \pm 3.2$ & $10.6 \pm 8.2$ & $<0.00 I^{*}$ \\
\hline Smoking history (pack-years) & $31(15)$ & $35(26)$ & $40(21)$ & 0.08 \\
\hline
\end{tabular}

Notes: Data is presented as median (interquartile range), unless otherwise noted. § Variables without Gaussian distribution; $P$-value was calculated using Kruskal-Wallis test. *Differences are significant. ${ }^{*}$ Variables with Gaussian distribution; $P$-value was calculated using unpaired Student’s $t$-test.

Abbreviations: IKE, isometric knee extension; AECOPD, acute exacerbation of COPD; 6MWD, 6-minute walking distance; SLS, Single Leg Stance; TUG, Timed UP and Go; BBS, Berg Balance Scale; pts, points; FES, Falls Efficacy Scale; hs-CRP, high-sensitive C-reactive protein. 
Table 3 Correlation matrix presenting the association between respiratory volumes and quadriceps contraction strength

\begin{tabular}{llllll}
\hline & FVC (L) & FVC (\%) & FEV (L) & FEV $_{1}(\%)$ & FEV, $/$ FVC \\
\hline IKE left & $0.620^{* *}$ & $0.55 I^{* *}$ & $0.573^{* *}$ & $0.618^{* *}$ & $0.506^{* *}$ \\
IKE right & $0.636^{* *}$ & $0.559^{* *}$ & $0.619^{* *}$ & $0.626^{* *}$ & $0.565^{* *}$ \\
\hline
\end{tabular}

Notes: The strength of the correlations was assessed using Spearman's correlation coefficient. ${ }^{* *}$ Correlations are significant at 0.01 level.

Abbreviations: FVC, forced vital capacity; FEV , forced expiratory volume in I second; IKE, isometric knee extension.

impairment (BBS, SLS) in COPD patients. Other researchers showed that particularly when these biomarkers are elevated, muscle wasting becomes a serious complication. ${ }^{18}$ This could be a contributor factor for fatigability and reduced muscle endurance observed in COPD patients.

Moreover, there are studies which demonstrated that inhaled corticosteroids may also modulate systemic inflammation (corticosteroids users had CRP levels $40 \%$ lower than corticosteroids nonusers). ${ }^{18}$ If we take into consideration medication as a risk factor for falls, there are some concerns regarding corticosteroids, which may lead to peripheral muscle weakness. The use of corticosteroid therapy in COPD population has been estimated to be $\sim 61.5 \%$ for inhaled and $\sim 8.3 \%$ for oral corticosteroids. ${ }^{19}$ But in the cases we studied, the use of inhaled corticosteroids was much reduced and the quantity/ day was difficult to assess. Corticosteroid therapy interferes with the production of contractile proteins, and they increase intracellular proteolysis. All these may result in reduced muscle mass and strength in these patients. These observations suggest that systemic inflammation could be one of the missing links between balance impairment, muscle weakness, and COPD.

Even if none of our patients were on long-term oxygen therapy, we recorded significantly decreased $\mathrm{PaO}_{2}$ in AECOPD. Patients with severe COPD may have a decreased hemoglobin oxygen saturation level, which may result in local tissue hypoxia, muscle wasting, and weakness. Just 8 weeks at altitudes greater than $5,000 \mathrm{~m}$ has been shown to cause $10 \%$ reduction in muscle mass and peak power. ${ }^{6}$ Hypoxia may also induce inflammation, causing muscle atrophy. ${ }^{6}$ However, hypoxia itself cannot fully account for all the observed changes in skeletal muscle because many patients with COPD suffering from muscle wasting and dysfunction do not exhibit hypoxemia. ${ }^{6}$ Some studies had shown the negative effects of exacerbations and inflammatory status on cognitive function. In one of our study using Montreal Cognitive Assessment questionnaire test, we concluded that COPD patients present significant impairment of the cognitive status, especially in acute exacerbation and advance stages of the disease. Increased inflammatory markers in these patients are associated with the decrease of the cognitive functions. ${ }^{17}$ Previously, Dal Negro et a $\mathrm{l}^{15}$ and Dodd et a $\mathrm{l}^{16,20}$ obtained similar results. Roig et al in a prospective study performed on older adults, showed that people with cognitive impairments fell five times more often than people without them. ${ }^{19}$

Beauchamp et al in a comprehensive study performed on COPD patients, concluded that $46 \%$ of patients experiencing a fall/year and the standard clinical balance measures (BBS and TUG) discriminate self-reported fallers from nonfallers. Moreover, impaired balance confidence and the use of supplemental oxygen were significant predictors of falls in this population. ${ }^{2}$ We obtained similar results on all these tests: KE peak torque - an equivalent for IKE, BBS, TUG, 6MWD, and $\mathrm{ABC}$ (an equivalent for FES-I test).

In comparison to us, her COPD groups were divided into fallers and nonfallers. Their results showed that fallers had a higher TUG score than nonfallers and lower BBS, 6MWD, and $\mathrm{ABC}$ scores. Interestingly, even if the use of supplemental oxygen was higher in fallers ( $72 \%)$ vs nonfallers (24\%), the $\mathrm{FEV}_{1}(\%$ predicted) was higher in fallers $(42.7 \%)$ than in nonfallers $(40.6 \%){ }^{21}$

It is well known that the most important risk factor that leads to COPD is smoking. All our COPD patients in this study were smokers, so we assume that the inflammatory cytokines released over the years may lead to systemic inflammation. But in order to exclude a confounder, we also selected smokers for the control group.

Table 4 Correlation matrix presenting the association between inflammatory markers and quadriceps contraction strength and balance tests

\begin{tabular}{llllllll}
\hline & IKE left & IKE right & 6MWD & SLS & TUG & BBS & FES \\
\hline Fibrinogen & -0.230 & -0.212 & $-0.321^{*}$ & -0.266 & $0.378^{*}$ & -0.230 & $-0.384^{*}$ \\
hs-CRP & -0.218 & -0.174 & $-0.413^{* *}$ & $-0.423^{* *}$ & $0.449^{* *}$ & -0.285 & $0.50 I^{* *}$ \\
\hline
\end{tabular}

Notes: The strength of the correlations was assessed using Spearman's correlation coefficient. *Correlations are significant at 0.05 level. **Correlations are significant at 0.01 level.

Abbreviations: IKE, isometric knee extension; 6MWD, 6-minute walking distance; SLS, Single Leg Stance; TUG, Timed Up and Go; BBS, Berg Balance Scale; FES, Falls Efficacy Scale; hs-CRP, high-sensitive C-reactive protein. 
Certain limitations must be taken into consideration when interpreting our results. We could not precisely record the amount of inhaled corticosteroids used/patient. Falls were also recorded retrospectively based on recall and, therefore, could have underestimated the incidence of falls in our samples; also, we cannot precisely know the etiology of each event. Our small sample size limits the reliability of our logistic regression, eg, the mismatch between the number of falls related to aging inside each group.

\section{Conclusion}

This study has shown that COPD patients have an increased risk of falls, balance impairment, and lower extremity muscle weakness, comorbidities in which systemic inflammation may play an important part. Simple clinical measures of balance like BBS and IKE can predict the risk of falls in this population. Future research is needed to elucidate the mechanism underlying balance deficits in this population and to adjust fall prevention strategies, given the devastating consequences of this problem.

\section{Acknowledgments}

The authors would like to thank Alexandru F Crisan for excellent assistance with the collection of data and Butiu Lia for the professional linguistic review.

\section{Disclosure}

The authors report no conflicts of interest or any financial interests related to the research. The authors alone are responsible for the content and writing of the article.

\section{References}

1. Ries AL, Bauldoff GS, Carlin BW, et al. Pulmonary rehabilitation: joint ACCP/AACVPR evidence-based clinical practice guidelines. Chest. 2007; 131(5 suppl):4S-42S.

2. Beauchamp MK, Hill K, Goldstein RS, Janaudis-Ferreira T, Brookset D. Impairments in balance discriminate fallers from non-fallers in COPD. Respir Med. 2009;103:1885-1891.

3. Guideline for the prevention of falls in older persons. American Geriatrics Society, British Geriatrics Society and American Academy of Orthopaedic Surgeons Panel on Falls Prevention. J Am Geriatr Soc. 2001; 49(5):664e72.

4. Soriano TA, DeCherrie LV, Thomas DC. Falls in the community-dwelling older adult: a review for primary-care providers. Clin Interv Aging. 2007; 2(4):545-553.

International Journal of COPD

\section{Publish your work in this journal}

The International Journal of COPD is an international, peer-reviewed journal of therapeutics and pharmacology focusing on concise rapid reporting of clinical studies and reviews in COPD. Special focus is given to the pathophysiological processes underlying the disease, intervention programs, patient focused education, and self management protocols.
5. Rubenstein LZ, Josephson KR, Robbins AS. Falls in the nursing home. Ann Intern Med. 1994;121:442-451.

6. Wüst RC, Degens H. Factors contributing to muscle wasting and dysfunction in COPD patients. Int J COPD. 2007;2(3):289-300.

7. Wouters EF. Chronic obstructive pulmonary disease. 5 : systemic effects of COPD. Thorax. 2002;57:1067-1070.

8. Sin DD, Jones RL, Mannino DM, Paul Man SF. Forced expiratory volume in 1 second and physical activity in the general population. $\mathrm{Am}$ J Med. 2004;117:270-273.

9. Rabe KF, Hurd S, Anzueto A, et al; Global Initiative for Chronic Obstructive Lung Disease. Global strategy for the diagnosis, management, and prevention of chronic obstructive pulmonary disease: GOLD executive summary. Am J Respir Crit Care Med. 2007;176(6): $532-555$.

10. Yardley L, Beyer N, Hauer K, Kempen G, Piot-Ziegler C, Todd C. Development and initial validation of the Falls Efficacy Scale-International (FES-I). Age Ageing. 2005;34(6):614-619.

11. Berg KO, Wood-Dauphinee SL, Williams JI, Maki B. Measuring balance in the elderly: validation of an instrument. Can J Public Health. 1992;83(suppl 2):S7-S11.

12. Springer BA, Marin R, Cyhan T, Roberts H, Gill NW. Timed Up and Go (TUG), Minnesota falls prevention. Normative values for unipedal stance test with eyes opened and closed. J Geriatr Phys Ther. 2007; 30(1):8-15.

13. ATS Committee on Proficiency Standards for Clinical Pulmonary Function Laboratories. ATS statement: guidelines for the six-minute walk test. Am J Respir Crit Care Med. 2002;166:111-117.

14. Kim WK, Kim DK, Seo KM, Kang SH. Reliability and validity of isometric knee extensor strength test with hand-held dynamometer depending on its fixation: a pilot study. Ann Rehabil Med. 2014;38(1): 84-93.

15. Dal Negro RW, Bonadiman L, Tognella S, Bricolo FP, Turco P. Extent and prevalence of cognitive dysfunction in chronic obstructive pulmonary disease, chronic non-obstructive bronchitis, and in asymptomatic smokers, compared to normal reference values. Int J COPD. 2014;9: 675-683.

16. Dodd JW, Getov SV, Jones PW. Cognitive function in COPD. Eur Respir J. 2010;35:913-922

17. Crişan AF, Oancea C, Timar B, Fira-Mladinescu O, Crişan A, Tudorache V. Cognitive impairment in chronic obstructive pulmonary disease. PLoS One. 2014;9(7):e102468.

18. Sin DD, Man SFP. Skeletal muscle weakness, reduced exercise tolerance, and COPD: is systemic inflammation the missing link? Thorax. 2006;61:1-3.

19. Roig M, Eng JJ, MacIntyre DL, Road JD. Falls in patients with chronic obstructive pulmonary disease: a call for further research. Respir Med. 2009;103(9):1257-1269.

20. Dodd JW, Chung AW, van den Broek MD, Barrick TR, Charlton RA, Jones PW. Brain structure and function in chronic obstructive pulmonary disease. A multimodal cranial magnetic resonance imaging study. Am J Respir Crit Care Med. 2012;186(3):240-245.

21. Beauchamp MK, Brooks D, Goldstein RS. Deficits in postural control in individuals with COPD- emerging evidence for an important secondary impairment. Multidiscip Respir Med. 2010;5(6):417-421.

This journal is indexed on PubMed Central, MedLine and CAS. The manuscript management system is completely online and includes a very quick and fair peer-review system, which is all easy to use. Visit http://www.dovepress.com/testimonials.php to read real quotes from published authors. 